\title{
Transgression, rites de rajeunissement et mort culturelle du roi chez les Jukun et les Rukuba (Nigeria central)
}

Transgression and Rites for Rejuvenating the King and Culturally Taking His Life among the Jukun and Rukuba (Central Nigeria)

Jean-Claude Muller

\section{OpenEdition \\ Journals}

Édition électronique

URL : http://journals.openedition.org/span/865

DOI : $10.4000 /$ span. 865

ISSN : 2268-1558

Éditeur

École pratique des hautes études. Sciences humaines

Édition imprimée

Date de publication : 1 décembre 1990

Pagination : 49-68

ISSN : 0294-7080

Référence électronique

Jean-Claude Muller, «Transgression, rites de rajeunissement et mort culturelle du roi chez les Jukun et les Rukuba (Nigeria central) », Systèmes de pensée en Afrique noire [En ligne], 10 | 1990, mis en ligne le 17 juin 2013, consulté le 19 avril 2019. URL : http://journals.openedition.org/span/865 ; DOI : $10.4000 /$ span.865 


\title{
DU ROI CHEZ LES JUKUN ET LES RUKUBA (NIGERIA CENTRAL)'
}

\author{
par \\ Jean-Claude Muller
}

La carrière des rois sacrés, que Frazer appelait "divins", est marquée par trois caractéristiques principales. La première se manifeste lors de l'intronisation du souverain qui ne devient véritablement roi qu'au prix d'une transgression qui le rend ambivalent, à la fois bon et mauvais, responsable de la prospérité comme des catastrophes majeures qui affectent son peuple. La seconde est que le roi doit, à intervalles fixes mais variables selon les sociétés, subir des rites de rajeunissement (rejuvenation rites) qui lui permettent de continuer à assumer sa tâche. Ces rites prennent souvent la forme d'un sacrifice où le roi est tué par procuration, un substitut en tenant lieu. La troisième caractéristique est que le roi ne peut mourir de mort naturelle: on le met à mort soit à date fixe, soit lorsqu'il veillit, ou encore lorsqu'il ne remplit pas ses devoirs et que des catastrophes révèlent qu'il n'est plus apte à gouverner. Ces trois caractéristiques de base sont susceptibles d'être interprétées différemment et par les ethnologues et par les populations concernées, ce qui fait qu'une

\footnotetext{
'Le travail de terrain chez les Rukuba s'est déroulé de janvier 1964 à septembre 1967, dans le cadre d'une mission de l'Unesco à Jos; de mai à septembre 1968 et de novenbre 1971 à février 1972, ces deux derniers séjours étant respectivenent financés par l'Université de Rochester, N.Y., et le Conseil des Arts du Canada.
} 
interprétation généralisante à partir d'un cas particulier peut fort bien être réfutée par les données d'un autre cas particulier, comme l'a si bien montré récemment Adler (1987). Néanmoins, ces trois caractéristiques communes demeurent et il nous semble que des comparaisons cas par cas dans des régions relativement circonscrites permettent de montrer leurs variations et transformations, indépendamment d'une interprétation globalisante du phénomène.

Les deux populations que nous avons choisi de comparer sont les Jukun et les Rukuba du Nigéria central. Les Jukun ont été pendant longtemps, grâce à l'étude de Meek (1931), un des prototypes de la royauté "divine" dans la littérature anthropologique. Ils furent les fondateurs d'un royaume (fort important dans la vallée de la Bénoué jusqu'au XVIII" siècle) qui avait assujetti nombre de populations à partir de la capitale, Kororofa, et de garnisons satellites établies aux marches du royaume, Pindiga, Gwana, Donga, Wase, Wuru, Kona, Takum et Abinsi. Ces groupes satellites existent toujours aujourd'hui, mais la capitale déménagea à Wukari après la défaite des Jukun par les Peul au XIX siècle. Le système de filiation des Jukun sera examiné plus loin car il pose des problèmes épineux pour notre propos.

Les Rukuba, quant à eux, sont situés sur le Haut Plateau nigérian. Patri-virilocaux, ils sont répartis dans plus de vingt villages; chacun est doté d'un chef, mais deux villages effectuent alternativement pour tous les autres les rites les plus importants de la chefferie et, tout comme les petits royaumes satellites jukuns sont dépendants de Wukari, les villages rukuba reconnaissent l'autorité rituelle alternée de ces deux villages.

Ces deux peuples ne se connaissaient pas jadis ${ }^{2}$ mais ils font partie d'un même ensemble culturel et nous montrerons que leurs rites royaux respectifs sont des variations l'un de l'autre. L'on retrouve ici les

\footnotetext{
'2 Les Jukun, au plus fort de l'expansion de leur ropaurre, au XVIIIe siècle, ont contrôlé une région assez vaste au sud-est du territoire rukuba. Jukun et Rukuba présentent incontestablement des similitudes culturelles (notamment en ce qui concerne leur conception de la royauté sacrée) mais leurs langues sont fort différentes. La langue jukun appartient au groupe B (jukunoid) des langues Benue-Congo de la classification de Greenberg (1966) alors que les Rukuba font partie du groupe A des langues du Plateau, sous-groupe 4, appartenant également à la famille Benue-Congo.
} 
difficultés soulignées par Dumézil: on peut analyser deux transformations d'une structure sans pouvoir dire si l'une dérive de l'autre ou si toutes deux sont des variantes d'une troisième que l'on ne sait où situer dans l'espace ou dans le temps. La parenté des rituels jukun et rukuba réside dans les similarités et les différences d'une structure comportant les trois caractéristiques de base déjà mentionnées dont nous allons analyser les transformations: les transgressions, les rites de rajeunissement, la mort culturelle du roi.

\section{Les transgressions}

Examinons d'abord les transgressions que l'on impose au roi jukun. La question n'est pas simple car les Jukun ont plusieurs rois qui reconnaissent tous comme souverain celui de Wukari. Celui-ci est considéré comme le plus important et il entreprend les rites les plus élaborés. C'est d'ailleurs à son propos que nous possédons les renseignements les plus riches, et sauf mention contraire, notre analyse portera sur les données relatives au roi de Wukari.

Après qu'un conseil d'anciens a désigné, dans la famille royale, celui qui va être intronisé, le candidat est attrapé, dévêtu puis rhabillé avec les insignes de son office (Meek 1931: 133). Il entre en réclusion pour quelques jours, sept à Pindiga et à Gwana, cinq à Wuru, parce que, suggère Meek (1931: 135), les esprits sont échauffés en temps d'élection et il vaut mieux que le roi reste dans son palais). Après son intronisation, on donne à manger au roi, sans qu'il le sache et en grand secret, une partie du coeur séché et réduit en poudre de son prédécesseur ainsi que les fluides recueillis sur son cadavre lors de sa momification. Le reste de la poudre de coeur est périodiquement mêlé à la nourriture du roi. Meek (1931: 168) écrit que les Jukun du Ghana mangent le coeur de leurs ennemis pour obtenir "complete possession of their life-soul". Dans le cas du roi, l'anthropophagie est d'une autre nature; elle est de l'ordre de la transgression. En effet, les Jukun ne pratiquent pas le cannibalisme intra-ethnique. C'est pour s'approprier les vertus des ennemis tués au combat que les 
guerriers mangent le coeur de leurs ennemis, alors que le roi ingère une partie de son prédécesseur. Un acte aussi dangereux ne peut être réservé qu'à un seul homme. Meek n'explique pas le sens de cet usage; il le met sur le même plan que tous les autres rites qui rendent le roi sacré, sans plus de précisions. Mais nous allons lui accorder une attention toute particulière.

Examinons à présent le domaine de la vie sexuelle. Le roi commet, apparemment, une autre transgression. Il se met nu devant une des épouses de son prédécesseur; il ne pourra avoir de relations sexuelles avec elle que durant deux nuits, mais elle devient son épouse principale (Meek 1931: 138-139). L'interprétation de cette coutume par Meek est intéressante à noter: "Mr Hocart has recently drawn attention to the identity of installation and marriage, the underlying idea being, perhaps, that marriage is necessary to installation just as a wife is necessary to creation. There may be the further idea that by marrying a widow of the late king the new king, if of a different dynasty, legitimizes the succession. It was common in ancient Egypt for the founder of a new dynasty to marry the widow of the last king". Nous n'avons pas assez de précision pour dire si cette union rituelle doit être considérée comme un inceste, ainsi que le pense Luc de Heusch (1958: 128-130; 1987: 122-124). Les Jukun étaient matrilinéaires mais, à l'époque des recherches sur le terrain de Meek, ils étaient en train d'adopter les principes de la patrilinéarité et Meek préfère qualifier leur système de bilatéral. Dans les régions jukun plus enclines à conserver la matrilinéarité, Meek (1931: 107) décrit ainsi l'héritage des veuves : "that is to say that widows are heritable by brothers and sisters'sons, sons being explicitely excluded". Mais la situation est plus compliquée car Meek affirme clairement que la succession au trône se fait en ligne patrilinéaire. Dans ce cas, le roi jukun serait tout simplement l'héritier d'une des femmes du roi précédent. Ce serait un héritage agnatique, comme le pratiquent (hormis pour la propre mère) plusieurs populations de la région. Contrairement à ce qui se passe ailleurs en Afrique, il ne s'agirait pas d'un inceste symbolique. 
Meek (1931: 115) nous dit aussi que chez les Jukun, l'idée de recevoir en héritage une jeune veuve d'un grand-père serait considérée avec horreur. En outre, l'héritage des veuves d'un oncle paternel est tabou dans la plupart des communautés car on l'assimile à un mariage avec la propre mère. Meek affirme que ces interdits s'appliquent à la plupart des communautés jukun, sans préciser lesquelles. Puisque la succession est patrilinéaire dans la dynastie jukun, il est clair, si l'on suit Meek, que le roi de Wukari ne peut qu'être un frère, un fils, un petit-fils ou un neveu paternel d'un roi précédent; dès lors en s'unissant à l'une des veuves de son prédécesseur, il se placerait dans la catégorie des transgresseurs. Mais, toujours selon Meek, ces mariages par héritage auraient été de règle dans certains groupes (probablement patrilinéaires). Ils ne constitueraient donc pas une exception royale, ce qui nous laisse perplexe encore une fois.

Pour ajouter encore à la confusion, les Jukun prétendent fermement que la royauté a toujours été transmise en ligne patrilinéaire : l'on dit que si un neveu utérin accède au trône, les lions envahiront la ville (Meek 1931: 37). Mais Meek (1931: 61-62) se montre sceptique et donne d'assez plausibles raisons pour penser que c'est la conquête peul qui a provoqué le passage de la matrilinéarité à la patrilinéarité, les Peul refusant des vassaux dont le régime successoral serait matrilinéaire. Comme nous l'avons dit plus haut, de nombreux groupes jukun autorisent l'héritage patrilinéaire des veuves et il serait bien étrange que les clans royaux, qui observent strictement les principes patrilinéaires, interprètent cet usage comme un inceste.

Il faut encore tenir compte d'une autre donnée plus récente qui clarifie le problème laissé en suspens par Meek. Young (1966: 140) nous assure que deux lignages royaux avaient des droits alternatifs sur le trône; cette alternance aurait été pratiquée assez régulièrement (fairly consistently). Seuls les fils d'anciens rois étaient éligibles. On se souviendra que l'épouse principale du roi, qui est écartée de sa couche après deux nuits, est une veuve de son prédécesseur; elle provient donc de la lignée alterne. On ne voit vraiment pas dans ce cas ce qu'il y aurait d'incestueux dans cette union rituelle. Notre tâche 
se trouve dès lors simplifié: si l'on suit Young, il n'y aurait donc pas d'inceste'.

Penchons nous maintenant plus en détail sur les autres femmes qui entourent le roi. Et d'abord sur cette reine classificatoire que Meek appelle la Angwu Tsi. Meek (1931: 324-325 et 340) dit d'elle : "At the present time, the angwu tsi may be any old woman of royal lineage, but in the past, il was customary to appoint as angwu tsi one of the widows of a former king..." et "...She must on appointment have passed the age of menstruation and under no circumstances may she have sexual intercourse". D'autres précisions sont ajoutées : "..the angwu tsi is regarded almost as a queen, the female counterpart of the king. She is commonly spoken of as "the wife of the king", but she does not live in the king's palace, nor does she have marital relations with the king". Ceci semble clore le problème de l'inceste... Mais le dernier chercheur - à notre connaissance - à avoir travaillé chez les Jukun, Masao Yamaguchi (1974: 22) qui appelle cette reine officielle awun tyi, prétend que le roi a des rapports sexuels avec elle alors que Meek nous dit expressément qu'il n'en est rien. Ceci n'est pas pour éclairer notre lanterne... Inceste réel ou symbolique ? Difficile de trancher.

Le roi a aussi une soeur officielle, la angwu kaku, qui est la fille d'un ancien monarque. Irstam (1944: 174) suggère qu'elle aurait des relations sexuelles avec le roi mais on n'en trouve aucune trace dans le livre de Meek sur lequel Irstam base son interprétation. Pas plus que pour la wakuku, on ne peut parler d'inceste. Chacune de ces femmes remplit une fonetion particulière. La wakuku et la angwu tsi rappellent étrangement, comme le fait remarquer Luc de Heusch (1958: 128-130, 1987: 122-124), les "petites mères" des rois nyoro; toutes deux doivent être ménopausées et donc d'un certain âge. Cependant, elles ne sont pas appelées mères mais épouses.

Meek mentionne encore de manière explicite un office disparu, rempli par la kushegê. Celle-ci était nommément une mère officielle du roi

\footnotetext{
${ }^{3}$ Nous citons Young plutôt que Meek car le premier a établi une liste de rois qui, malgré quelques inconsistances, semble montrer que l'alternance fut respectée.
} 
mais son statut - veuve d'un prédécesseur? mère classificatoire? - n'est pas spécifié. On ne sait pas non plus si le roi avait des relations sexuelles avec elle. Cette ancienne fonction a peut-être été remplacée par celle de l'angwu tsi, car Yamaguchi (1974: 9 et 12) parle de celleci comme d'une reine mère.

S'il n'est pas certain que les rois jukun commettent l'inceste, en revanche, le rituel d'installation comprend un autre type de transgression: le cannibalisme rituel. Celui-ci semble bien, dans cette région, prendre le relais de l'inceste symbolique ou réel. Chez les Mbum (Meek 1931a II: 493), les Yoruba d'Oyo (Morton-Willams 1967: 53-54), à Ifé (Parrinder 1956: 15) et chez les Ekoi (Jeffreys 1939: 101-102) les souverains mangent le coeur de leurs prédécesseurs comme les rois jukun.

Si nous nous tournons vers les transgressions commises par les chefs rukuba, nous restons toujours dans le registre du cannibalisme. Il n'y a pas l'ombre d'un inceste chez les Rukuba, bien que le chef ait des assistantes jouant des rôles rituels qui, toutefois, sont bien moins élaborés que ceux réservés aux épouses royales jukun.

Dans la mesure du possible, le chef est choisi par les anciens du lignage des faiseurs de rois. Les lignées alternent parfois. Ils désignent toujours un homme jeune de ce groupe de descendance patrilinéaire et ils ne peuvent en aucun cas assumer eux-mêmes les fonctions de chef. Les critères de sélection sont nombreux et je n'y reviendrai pas ici (Muller 1975, 1980: 151-153). Ce qui importe, c'est que le chef soit le fils d'une épouse préférentielle du clan du chef. Cette épouse est une nièce utérine du village du chef, ce qui fait de son fils, outre un agnat du village par son père, un petit-neveu utérin dans son village, un statut structuralement ambigu puisque les prohibitions qu'il doit respecter comme agnat sont en même temps levées de par son statut d'utérin qui lui permet d'enfreindre ces tabous. Je me bornerai à rappeller ici que le chef combine deux statuts familiaux antithétiques.

L'homme désigné pour cette fonction est "attrapé" : on lance sur lui une corne de céphalophe dans laquelle on a mis quelques restes du corps de son prédécesseur - peau des lèvres, ongle du pouce droit 
et ongle du gros orteil du pied gauche - ce qui le "tue" métaphoriquement. Il se sauve chez son oncle maternel où il restera sept jours à porter le deuil de son ancienne identité. Le septième jour, il s'habille comme un initié à la fỉn de son initiation et rentre chez lui; on lui donne à boire une calebasse de bière dans laquelle trempe la calotte crânienne d'un des chefs précédents. Ces restes funèbres sont très puissants et tueront le chef s'il n'est pas assez "fort", ou le feront disparaître avant la fin de sept ans de règne. Au contraire, si le chef est adéquat, ces ossements contribueront à le rendre gros et gras .

Cette intronisation est pratiquée partout, mais les deux villages rituellement les plus importants, Ishe et Uniu, l'enrichissent d'une particularité remarquable. Le jour de l'intronisation, on tue un bébé du clan du chef, lui-même chef potentiel; il subit d'ailleurs les mêmes rites funéraires qu'un vrai chef. En outre, on prélève sur son corps quelques morceaux de chair qu'on mêle à la viande d'un bélier sacrifié et c'est ce mélange qu'on donnera à consommer au chef sans qu'il en connaisse la nature. Il absorbe donc la sagesse de ses prédécesseurs et la vigueur de son successeur potentiel, ajoutant ainsi une génération de plus à sa propre vie, ce qui donne naissance à la croyance rukuba qu'un chef est censé mourir vieux, même très vieux. En effet, la caractéristique d'un grand chef est précisément qu'il meurt à un âge avancé.

Le corps du bébé sacrifié est séché au soleil, après avoir été vidé de ses entrailles, enterrées à part. Il se transforme en fait en une momie que l'on garde précieusement, nous verrons bientôt pourquoi. L'efficacité rituelle de l'absorption de la chair du jeune enfant rejaillit sur le destin de tous les autres chefs de village: tous les Rukuba partagent l'idéologie des longs règnes.

C'est bien d'une transgression qu'il s'agit ici. L'endo-cannibalisme est strictement prohibé chez les Rukuba: ceux-ci mangeajent sélectivement les membres de certaines ethnies ennemies mais s'abstenaient rigoureusement de consommer les ressortissants des tribus dites amies, les amis et les ennemis n'étant pas nécessairement les mêmes d'un village à l'autre. Le chef est le seul à transgresser cette règle. 
Nous avons dit que lorsqu'il revient de chez son oncle maternel pour subir les rites d'intronisation, il est vêtu comme un initié à la fin de son initiation; c'est dans cet accoutrement qu'il boit la bière dans laquelle baigne la calotte crânienne d'un de ses anciens chefs. Lors de leur initiation (obligatoire pour tous avant l'âge de douze ans), les Rukuba se voient présenter la même calebasse mais ils refusent ostensiblement d'en boire la bière (Muller 1989: 141-149). Le chef est donc, comme le roi jukun, le seul transgresseur. Les Rukuba reconnaissent explicitement que cette pratique d'endo-cannibalisme est un acte abhorré. Cette transgression est, croit-on, 1'un des secrets les mieux gardés.

Les Rukuba interprètent aussi cet acte rituel comme un test, une sorte d'examen à passer. Si le chef est assez "fort", s'il a la chance avec lui, en un mot s'il est "blanc", les ossements des anciens chefs le rendront gras et prospère. Si, au contraire, il est trop faible pour sa tâche, son règne ne dépassera pas sept ans; les ossements le tueront. Mais, s'il passe le cap des sept premières années sans encombre, les Rukuba sont d'avis qu'un règne très long, un "vrai" règne, commence. Ces sept premières années sont des années probatoires. Ce n'est qu'après cette période qu'on commence vraiment à prendre un chef au sérieux. Mais il ne devient vraiment prestigieux que s'il a vu, depuis sa naissance, se dérouler sept rituels de rajeunissement, kugo. Ceux-ci ont lieu, en théorie, tous les quatorze ans. Un grand chef devrait donc être centenaire. Il est vrai que kugo se fait en réalité tous les dix ans environ, ce qui rabaisse l'âge requis à soixante-dix ans. Les Rukuba se souviennent très bien de chefs très vieux ${ }^{\wedge}$. On comprend qu'un chef est censé devenir une sorte de Mathusalem ( $s i$ tout va bien pour lui) puisqu'il réunit en lui la sagesse de ses prédécesseurs et la jeunesse de son successeur potentiel dont il a mangé la chair lors de son intronisation.

Sous des formes diverses, la transgression transforme le roi sacré en un être ambivalent, bénéfique et redoutable à la fois. Nous allons

\footnotetext{
4 Ace, le chef du village de Kissagyp, régnait déjà en 1905 à l'arrivée des Anglais; il était toujours en fonction en 1968. Il mourut vers 1970, à plus de quatre-vingt-dix ans.
} 
voir par la suite que la bonne et la mauvaise part du roi sont l'objet d'un traitement différentiel lors des rites de rajeunissement.

\section{Les rites de rajeunissement}

Chez les Jukun, le roi était mis à mort tous les sept ans et cette croyance est encore partagée par les habitants des régions éloignées de la capitale qui calculent leur liste de rois en les comptant de sept ans en sept ans (Young 1966: 150). Mais il semble bien que cette règle n'a plus été respectée depuis quelque deux cents ans car on pouvait prolonger le règne du roi en le soumettant à un rite, appelé ando $k u$, que décrit Meek (1931: 139 ssq.). Cette cérémonie n'était plus effectuée à l'époque des recherches de Meek en 1927. Le roi Ashu Manu II avait tenté de restaurer le rituel mais il mourut tragiquement en 1871. Aucun de ses successeurs ne reprit la tradition. Ce rite de rajeuissement avait donc été abandonné depuis au moins soixante ans lors des enquêtes de Meek.

Selon l'une des versions recueillies, le roi en personne devait tuer un esclave d'un coup de lance. Selon une autre, il blessait seulement la victime, qui était achevée par un officiel qui utilisait la lance et le couteau royaux dont il avait par ailleurs aussi la garde. Le roi abandonnait ensuite son cheval et se sauvait en brousse où on le recherchait pour finalement le retrouver tout nu. On le rhabillait et, monté sur un cheval blanc, il était reconduit en ville au milieu des acclamations. Il recevait alors un nouveau nom. Meek, comparant cette cérémonie à celle des Ganda de l'Ouganda, estime que l'esclave représente le roi, mis à mort par personne interposée, ce qui permet au souverain de renaître et d'entamer une vie nouvelle.

Meek (1931: 165) ajoute encore que le rite ando ku était une sorte d'ordalie et qu'un roi qui ne donnait pas satisfaction était probablement tué lors de cette opération. Dans le cas contraire, il était épargné et autorisé à conserver le pouvoir, le rite de rajeunissement revenant au cours de la sixième année du nouveau règne. Il semble que l'acquisition d'un nouveau nom, après chaque ando ku réussi, correspondait 
à l'accession à une nouvelle dignité. Meek (1931: 142) en parle obliquement : "...the Jukun ceremony of Ando ku (...) for the prolongation of the life of the king would indicate that divine kings may advance from a lower to a higher grade". Toujours selon Meek (1931: 142), il était interdit aux rois de Wukari de regarder le site de l'ancienne capitale à moins qu'ils n'aient accédé à un titre plus élevé que Manu. Manu était un titre conféré par la communauté hausa de Wukari (Abakwariga) aux rois de cette ville. Ceci semble effectivement suggérer un changement de nom après chaque ando ku.

Les Rukuba, quant à eux, effectuent leurs cérémonies de rajeunissement, appelées kugos, non pas tout les sept ans mais tous les quatorze ans (ceci en théorie du moins car, en pratique, elles reviennent, comme nous l'avons dit, tous les dix ans environ). On sélectionne deux victimes substitutives: un vieillard et un bélier (appartenant au roi ou fourni par lui). Les Rukuba élèvent peu de moutons et le chef doit le plus souvent en acheter un pour la circonstance. Cependant, il faut que le mouton ainsi acquis passe au moins une nuit dans l'étable du chef pour que le lien d'appartenance soit bien marqué. On tue l'animal et on en donne la chair à manger au vieillard qui devient ainsi impur. On se souviendra que le roi sacré transgresseur est un personnage ambivalent. Le rituel va opérer un double transfert de la part maudite du roi. Celle-ci est d'abord communiquée au bélier sacrifié; elle se fixe ensuite sur le vieillard qui en a consommé la chair. On ne mettra pas à mort cet être qui est le véritable double du roi; on le laissera errer autour du village (où il ne peut pénétrer) quémandant sa nourriture par l'arrière des maisons. NuJ ne peut entrer en contact avec lui ni avec la calebasse qu'il utilise pour mendier. Selon certaines informations, il est censé mourir au cours des sept années suivantes; d'autres estiment qu'il disparaîtra au cours de la septième année. S'il ne meurt pas de mort naturelle au cours de cette période, certains disent que le rituel (qui est une entité per-

\footnotetext{
"Le terme ando ku signifie "la maison du roi", aku étant le terme pour roi; kuqo veut dire "le grand $\underline{k u}^{\prime \prime}, \underline{k u}$ étant le nom du rite sans qu'il ait une signification car on emploie toujours le termo kugo. Les deux termes ne sont pas linguistiquement parents.
} 
sonnifiée chez les Rukuba) ne manquera pas de le tuer, alors que d'autres prétendent que ce sont les assistants rituels du chef qui le mettent à mort à ce moment. On se souviendra ici que sept ans est le terme maximal du règne d'un mauvais chef. Les Rukuba insistent sur le fait que le vieillard impur et chassé du village dont il hante les alentours est comme un mauvais chef; il est véritablement le double du roi dont il incarne la part mauvaise. Si l'on compare à cet égard Rukuba et Jukun, on ne peut manquer de repérer ici une inversion du système symbolique: le mauvais roi rukuba règne sept ans, alors que l'idéologie jukun estime qu'un bon roi ne doit pas dépasser ce laps de temps.

Mais nous avons vu que dans la pratique, on prolongeait le règne des rois jukun grâce au rite ando ku. A y regarder de plus près, on s'aperçoit que cette prolongation est en fait une renaissance. Le roi, après avoir tué son double, se retrouve tout nu en brousse. Dans une séquence qui rappelle l'intronisation, on le rhabille et on lui donne un nouveau nom. C'est donc la totalité de la personne royale qui est sacrifiée sous la forme de son substitut, la bonne part comme la mauvaise. Il n'y a rien de semblable chez les Rukuba où seule la mauvaise part du chef se trouve expulsée avec la vieillard maudit. Les deux idéologies locales divergent significativement à ce propos. Il est clair que chez les Jukun on est en présence d'un nouveau roi alors que rien ne change chez les Rukuba. L'expulsion de la victime émissaire - représentant la mauvaise part du roi - est interprétée par ceuxci comme une remise en ordre du monde qui est gravement compromis.

Ces deux systèmes se présentent comme deux élaborations symboliques différentes gravitant autour du nombre sept: les rites de rajeunissement ont lieu tous les sept ans chez les Jukun, tous les quatorze ans chez les Rukuba. Ceci peut être justifié, à titre d'hypothèse, de la manière suivante: dans l'idéologie aussi bien que dans la pratique rukuba, deux rois coexistent physiquement pendant les sept années qui suivent le rite de rajeunissement; le bon roi règne et le mauvais hante les alentours du village où il accumule saleté et impuretés (il ne peut ni se laver ni se coiffer; il est hirsute et ne peut 
s'approcher des maisons pour mendier que par l'arrière, à l'endroit où l'on jette les détritus de la cuisine et du balayage et où l'on urine, endroit justement appelé aya, "déchets"). On peut présumer que les impuretés de la chefferie ne commencent à s'accumuler sur le roi règnant qu'après la mort de son double, cette accumulation nécessitant l'expulsion d'un nouveau double sept ans plus tard, soit exactement le nombre d'années que règne le roi jukun avant le rite de rajeunissement. Le roi jukun est tué sous la forme d'un esclave alors que les Rukuba ne font qu'expulser la part maudite du chef en la transférant à un vieillard qui l'assumera pendant sept ans, le temps normal d'un règne jukun. C'est la neutralisation des forces mauvaises par leur transfert sur le double du chef qui permet de doubler le temps effectif $\mathrm{du}$ règne $\mathrm{du}$ chef rukuba. Ceci explique que les rites de rajeunissement ont lieu cette fois tous les quatorze ans au lieu des sept ans requis par le règne d'un roi jukun.

On se souviendra que, chez les Jukun, Meek (1931: 164) analyse ando $k u$ comme une ordalie (ordeal); il suggère qu'il est possible qu'un roi qui ne satisfaisait pas ses subordonnés était mis à mort à cette occasion. On retrouve la même idée dans le rite d'expulsion du double mauvais du chef chez les Rukuba. On a vu que, lors de l'intronisation des chefs des deux villages les plus importants (du point de vue rituel), l'on tuait un chef potentiel, un bébé qui avait toutes les qualifications pour devenir chef plus tard et qu'on le momifiait en le faisant sécher. Lors du rite de rajeunissement, le vieillard qui sera expulsé par la suite est exhibé devant quelques chefs de village. On l'installe de force sous un auvent et l'on cache la momie de l'enfant dans les branches du linteau. Le petit corps a été abondamment oint d'huile et des gouttes tombent par terre. Les chefs sont conviés à entrer l'un après l'autre sous l'auvent. Ils n'y restent qu'un instant avant de ressortir mais si l'un ou l'autre est touché par une goutte d'huile, il sait qu'il mourra avant le prochain rite de rajeunissement. Il est censé disparaître dans l'intervalle de quatorze ans qui va suivre. En fait, ce que les chefs viennent contempler n'est autre que leur propre double menaçant. On a affaire ici à une inversion de l'ordalie 
jukun. Alors que le roi jukun règnant tue son substitut vivant, c'est son substitut momifié qui décide de la mort du chef rukuba.

\section{La mort culturelle du chef}

Venons en à la troisième caractéristique des rois "divins": leur mort naturelle. Le roi jukun est tué dans les circonstances suivantes: après sept années de règne ou encore lorsqu'il tombe de cheval, lorsqu'il enfreint ses devoirs rituels ou lorsqu'il est malade au point de ne plus pouvoir remplir ses devoirs rituels. Il est également sacrifié après une famine ou une sérieuse sécheresse. Quelques proches du roi mettent fin à ses jours par strangulation. Son corps est ensuite vidé; on en prend le coeur qu'on sèche et réduit en poudre; on enterre les poumons et les viscères avant de boucaner le corps. Meek (1931: 164-177), qui décrit la mort et les funérailles du roi en grand détail, nous apprend que la durée de fumigation du cadavre varie de quatre à six mois car on doit le mettre en terre au début de la saison des pluies "lorsque le millet a atteint une hauteur de près d'un pied" (Meek 1931: 169). Aucun roi jukun n'est donc censé mourir naturellement. Comme le remarque fort bien Young (1966: 150), la mise à mort au terme d'une sécheresse ou d'une famine excessive fait du souverain un bouc émissaire.

C'est cet aspect, masqué mais présent chez les Jukun, qui est constamment relaté et mis en acte chez les Rukuba. Cependant, ceuxci ne tuent pas leur chef lorsque le village est en proie à une catastrophe (sécheresse, invasion de sauterelles, famine, zizanies incessantes entre factions que le chef est incapable de réconcilier, épidémies particulièrement meurtrières, morts subites en nombre inhabituel, défaites à la guerre). Le chef est simplement expulsé du village et il devra se rendre chez son oncle maternel où il cessera de nuire. Après son expulsion, on nomme un autre chef qui sera immédiatement intronisé. L'ancien chef est exclu de sa communauté; ce n'est pas une mort physique qu'il subit mais une mort sociale. 
On ne tue pas un chef vieillissant, affaibli ou malade; on le laisse s'éteindre en paix pendant que ses assistants accomplissent ses tâches rituelles. Cependant, le traitement du cadavre d'un chef mort de vieillesse est tout autre que celui réservé aux morts ordinaires. Outre les parties prélevées sur le corps qui serviront à "attraper" son successeur, ainsi que nous l'avons décrit plus haut, on conserve souvent ses cheveux; on les accroche en touffes sur l'anse qui sert à transporter la calebasse sacrée du village où trempent les ossements de ses prédécesseurs. Le chef est le seul à pouvoir s'en servir. On enterre le corps le plus rapidement possible. Comme les chefs d'un même village sont inhumés dans une tombe collective, on profite de son ensevelissement pour en extraire la calotte crânienne du chef précédent ainsi qu'un des os de l'avant-bras qui sert d'anse à la calebasse sacrée.

Si le chef n'est pas mis à mort culturellement, ses deux substituts le sont à sa place. Nous avons vu que le vieillard expulsé comme le double mauvais de celui-ci doit mourir au plus tard la septième année de son errance. Certains disent que le rituel le tue à l'époque prescrite alors que d'autres soutiennent que les assistants rituels du chef font office de bourreaux. Quoi qu'il en soit, le premier substitut du chef, le bébé du même clan est réellement sacrifié lors de l'intronisation. On se souviendra qu'il est étouffé et réduit à l'état de momie; ces deux traits rappellent respectivement la strangulation du roi jukun et le boucanage de son cadavre.

Signalons cependant une difficulté. Si le roi "divin" ou son substitut doit mourir culturellement de main d'homme chez les Jukun et chez les Rukuba, c'est parce qu'il s'est rendu coupable d'une transgression. Cependant, on pourra objecter que le bébé traité rituellement par les Rukuba est innocent. Pourquoi donc le sacrifier ?

La solution apparaîtra en effectuant une nouvelle comparaison avec les Jukun. Nous avons vu que chez ces derniers le roi renaît comme un être nouveau après les rites de rajeunissement. On le retrouve en brousse entièrement nu, comme un nouveau-né; on lui fait subir à nouveau une partie des rites d'installation et, surtout, on lui donne un nouveau nom. C'est le roi dans la totalité de sa personne ambivalente 
qui est symboliquement éliminé. Lors des rites de rajeunissement rukuba, nous avons remarqué que seule la mauvaise part du roi était expulsée sous la forme du vieillard. Le bébé rukuba qui n'a commis aucune transgression peut être interprété comme la figure symbolique symétrique et inverse du vieillard qui, lors du kugoa, a mangé la mauvaise part du chef. Le sacrifice du bébé serait alors celui de la bonne part du chef. Ce que les Jukun réalisent en une seule opération sacrificielle, est dédoublé chez les Rukuba.

Cependant, les Rukuba jouent ici sur une ambiguïté qui permet aussi de dire que le bébé sacrifié est bien le double du chef, pris en sa totalité, avec sa bonne part et sa part maudite, comme le roi jukun. Les Rukuba mettent en oeuvre les principes d'identité et d'équivalence des générations alternées: le petit-fils est la réplique du grandpère. Le chef doit en principe mourir très vieux; s'il meurt prématurément, ses funérailles seront néanmoins accomplies comme s'il était mort très âgé.

Le bébé qu'on sacrifie après sa mort lui est structuralement identique, il est son alter ego. Cette petite victime est à la fois le même et l'autre; elle est la stricte réplique sociologique du vieux chef, son grand-père. Celui-ci aurait dû être sacrifié juste avant sa mort naturelle mais on l'exécute sous la forme du bébé juste après son trépas. On peut donc dire indifféremment que le sacrifice concerne seulement la bonne part du chef ou la totalité de son être (à la fois bon et mauvais ) puisque grand-père et petit-fils sont identiques. Mais si l'on considère que le bébé n'a rien d'un transgresseur, c'est la bonne part du chef que l'on sacrifie, la part maudite étant expulsée sous la forme du vieillard lors des rites de rajeunissement. L'ambiguïté rukuba fait double coup car les deux interprétations sont valides. Nous sommes ici au coeur d'une dialectique où jouent le réel (le meurtre du bébé) et le symbolique.

Examinons rapidement les victimes représentant les rois dans les rites de rajeunissement africains. On y trouve des esclaves (comme chez les Jukun) et des animaux. Les Rukuba sont plus sophistiqués; d'une certaine façon, ils sacrifient de vrais chefs. Le vieillard est le 
chef puisqu'il a mangé le substitut de celui-ci (le bélier qui représente le chef), et le bébé est aussi un vrai chef. C'est parce qu'ils choisissent des victimes plus proches du chef (si l'on peut dire), que les Rukuba peuvent épargner ce sort au chef véritable.

\section{Conclusion}

M. Young (1966) a bien montré que les explications fonctionnalistes de la mort culturelle du roi ne résistaient pas à l'examen des faits jukun. Il serait encore plus difficile d'appliquer cette théorie aux faits rukuba. Si la théorie frazérienne a bien décelé le mécanisme du bouc émissaire, elle est pourtant incapable d'en saisir la logique : en particulier, elle serait bien en peine de rendre compte du sacrifice du bébé chef potentiel chez les Rukuba. Cette mort est associée à une spéculation sur la longueur des règnes suscitant des rituels qui permettent au roi de vivre le plus longtemps possible. Les Rukuba semblent avoir dédoublé le modèle jukun ou, plus vraisemblablement, les Jukun et les Rukuba ont créé chacun pour leur part leurs propres variations à partir d'un modèle commun. L'un est plus sophistiqué que l'autre, mais tous deux découlent d'une même logique interne.

Cependant, celle-ci ne saurait nous expliquer les attitudes contrastées que les deux populations adoptent à l'égard de leurs souverains. Le livre de Meek est rempli de notations relatives à la reconnaissance que l'on a pour le roi, pour son travail et pour sa personne. Lorsqu'il sort de son palais, il est salué par des cris et des exclamations : "Notre grain ! Nos haricots ! Notre pluie ! Notre santé! Nos récoltes ! Nos richesses !" qui mettent l'accent sur la prospérité découlant de la personne du roi. Les Rukuba sont beaucoup plus modérés et ne chantent pas les louanges des chefs. Lorsqu'ils discutent de la longueur des règnes, ils passent presque toujours sous silence les aspects bénéfiques de la chefferie qu'ils tiennent, si l'on peut dire, pour acquis et qu'ils ne soulignent pas. Ils sont, par contre, très impitoyables pour les chefs qui n'ont pas régné longtemps et n'ont que mépris pour ceux qui ont été déposés comme si ceux-ci les avaient 
trahis. En établissant la liste des chefs de villages, j'ai parfois, mais rarement, entendu faire l'éloge de certains règnes et des bonnes récoltes qui en étaient résultées. Cependant, ces éloges apparaissaient dans le discours moins pour souligner la grandeur de ces chefs que pour dénigrer ceux qui avaient failli. Les Jukun mettent plutôt l'accent sur l'aspect bénéfique de la chefferie alors que les Rukuba insistent davantage sur l'aspect bouc émissaire. Les deux aspects sont présents dans les deux sociétés mais chacune a choisi, pour des raisons qui nous échappent, de mettre l'accent sur des pôles opposés.

Jean-Claude Muller Université de Montréal

\section{Références bibliographiques}

Adler A.

1987 "Royauté et sacrifice chez les Moundang du Tchad", in Sous le masque de l'animal. Essais sur le sacrifice en Afrique noire (Cartry M. éd.), Paris, PUF, 89-130.

Ballard J.A.

1971 "Historical Inferences from the Linguistic Geography of the Central Nigerian Middle Belt", Africa, XLI, 4, 294-305.

Greenberg J.H.

1966 The Languages of Africa, Bloomington, University of Indiana Press/Paris-La Haye, Mouton.

Heusch L. de

1958 Essais sur le symbolisme de l'inceste royal en Afrique, Bruxelles, Université Libre, repris dans Ecrits sur la royauté sacrée, Bruxelles, Ed. de l'Université de Bruxelles, 1987.

Irstam T.

1944 The King of Ganda. Studies in the Sacred Institution of Kingship, Stockholm, Université d'Uppsala. 
Jeffreys M.D.W.

1939 "Some Notes on the Ekoi", Journal of the Royal Anthropological Institute, LXIX, 1, 95-108.

Meek K.

1931 A Sudanese Kingdom. An Ethnographical Study of the Jukunspeaking Peoples of Nigeria, Londres, Trench, Trubner and Kegan Paul ( réimpression 1969, New-York, Negro Universities Press).

1931 Tribal Studies in Northern Nigeria, Vol. I et II, Trench, Trubner and Kegan Paul (réimpression 1976, Millwood, N. Y., Kraus Reprint).

Morton-Williams P.

1967 "The Yoruba of Oyo", in West African Kingdoms in the Nineteenth Century, D. Forde and P. Kaberry eds, Oxford, Oxford University Press for International African Institute, 39-69.

Muller J.C.

1975 "La royauté divine chez les Rukuba (Benue-Plateau State, Nigéria)", L'Homme, XV, 1, 5-27.

1980 Le roi bouc émissaire. Pouvoir et rituel chez les Rukuba du Nigéria Central, Paris, L'Harmattan/Québec, Serge Fleury.

1989 La calebasse sacrée. Initiations rukuba (Nigéria Central), Grenoble, La Pensée Sauvage/Montréal, Les Presses de l'Université de Montréal.

Parrinder E.G.

1956 Divine Kingship in Africa", Numen, 362, 111-121.

Yamaguchi $\mathrm{M}$.

1974 "La royauté et le symbolisme dualiste chez les Jukun du Nigéria", Journal of Asian and African Studies, VIII, 129 .

Young M.W.

1966 "The divine kingship of the Jukun: A re-evaluation of some theories", Africa, XXXVI, 2, 135-153. 